\title{
Estimation of hysteretic damping of structures by stochastic subspace identification
}

Bajric, Anela; Høgsberg, Jan Becker

Published in:

Mechanical Systems and Signal Processing

Link to article, DOI:

10.1016/j.ymssp.2017.11.042

Publication date:

2018

Document Version

Peer reviewed version

Link back to DTU Orbit

Citation (APA):

Bajric, A., \& Høgsberg, J. B. (2018). Estimation of hysteretic damping of structures by stochastic subspace identification. Mechanical Systems and Signal Processing, 105, 36-50.

https://doi.org/10.1016/j.ymssp.2017.11.042

\section{General rights}

Copyright and moral rights for the publications made accessible in the public portal are retained by the authors and/or other copyright owners and it is a condition of accessing publications that users recognise and abide by the legal requirements associated with these rights.

- Users may download and print one copy of any publication from the public portal for the purpose of private study or research.

- You may not further distribute the material or use it for any profit-making activity or commercial gain

- You may freely distribute the URL identifying the publication in the public portal 


\title{
Estimation of hysteretic damping of structures by stochastic subspace identification
}

\author{
Anela Bajrića,*, Jan Høgsberg ${ }^{\mathrm{a}}$ \\ ${ }^{a}$ Technical University of Denmark, DTU Mechanical Engineering, Niels Koppels All Building 403, 2800 Kgs. Lyngby, \\ Denmark
}

\begin{abstract}
Output-only system identification techniques can estimate modal parameters of structures represented by linear time-invariant systems. However, the extension of the techniques to structures exhibiting non-linear behavior has not received much attention. This paper presents an output-only system identification method suitable for random response of dynamic systems with hysteretic damping. The method applies the concept of Stochastic Subspace Identification (SSI) to estimate the model parameters of a dynamic system with hysteretic damping. The restoring force is represented by the Bouc-Wen model, for which an equivalent linear relaxation model is derived. Hysteretic properties can be encountered in engineering structures exposed to severe cyclic environmental loads, as well as in vibration mitigation devices, such as Magneto-Rheological (MR) dampers. The identification technique incorporates the equivalent linear damper model in the estimation procedure. Synthetic data, representing the random vibrations of systems with hysteresis, validate the estimated system parameters by the presented identification method at low and high-levels of excitation amplitudes.
\end{abstract}

Keywords: Output-only system identification, Non-linear restoring force, Hysteretic damping, Linearization, Relaxation damping, Random vibrations

\section{Introduction}

The validation of models for structures and machinery operating under random environmental and operational conditions is a crucial step for reliability and safety analysis. To such purpose, various system identification techniques [1-3] have been developed in order to estimate and validate the dynamic properties of the investigated structure, based on vibration measurements. However, the most commonly used output-only dynamic identification method [4] has been developed for linear time-invariant systems, and the extension of such to non-linear system parameter identification has still not received much attention.

\footnotetext{
${ }^{*}$ Corresponding author

Email address: bajric.anela@gmail.com (Anela Bajrić )
} 
The increasing complexitiy and flexibility of modern engineering structures give rise to nonlinearities in various forms, and often these become progressively more significant as the vibration amplitude increases. In this context, the target of the paper is to propose a parametric output-only identification methodology suitable for a single-degree-of-freedom oscillator driven by random excitation which exhibit hysteretic behavior. The detailed formulation of the nonlinear benchmark identification problem adressed is described in [5].

Hysteresis is usually triggered by high levels of dynamic loading that emphasize the nonlinear nature of dissipative and restoring forces, which depend on the history of the motion rather than instantaneous motion. The nonlinear nature of the forces is significant in hysteretic behavior observed in large scale structures, when subjected to high levels of dynamic loading. Other examples of hysteretic properties can be encountered in magnetic, ferromagnetic and ferroelectric materials which are found in vibration mitigation devices such as Magneto-Rheological (MR) dampers [6].

Identification methods of systems affected by hysteretic behavior classified as input-output techniques are discussed in [7]. These include approaches based on evolutionary algorithms [8, 9], Bayesian inference methods [10], Markov Chain Monte Carlo methods [11] and a recent frequency domain approach with blackbox optimization for nonlinear estimation [12]. The input-output methods are limited to structures for which the excitation is measurable. A different identification strategy is necessary for dynamically excited structures with unmeasured and uncertain excitation, often experienced in connection with large structures or machinery where no straightforward way allows the excitation to be measured.

A classical approach for estimation of stochastic dynamic systems is the Kalman filtering for a state estimation [13] or the realization theory for estimation of the system based on random response. The latter is incorporated in the time domain operational modal analysis methods [14], which are limited to linear and time-invariant systems. Extensions of the classical Kalman filtering approach enable joint input and state estimation at locations of interests of sparsely monitored dynamic structures $[15,16]$. The state prediction of structures can also be applied for structural health monitoring as in [17], which demonstrates that the statistical properties of the states can be regarded as a damage indicator. The joint state and input estimation for structural applications assume a known representative finite element model of a linear system, and are associated with a heavy computational burden when using the Kalman filter, which can be reduced by a model reduction scheme [18]. Moreover the joint state and input estimation is numerically unstable and results in unreliable input estimation when based on acceleration measurements [19], which are unfortunately often the only measurable signal available for a dynamic structure.

The numerical issues associate with observability and rank deficiency are treated in the augumented joint state-input estimation proposed in [20] and experimentally demonstrated in [21], also for a system with known model parameters. In [22-24] an online parametric identification based on Kalman filtering for state estimation is proposed for the non-linear hysteretic system. More recently [25] also proposed a joint state- 
parameter-input estimation method for linear time varying systems, which has the advantages of being able to cope with mode veering effects. It has been applied in [26] for tracing the time varying stiffness properties of an offshore wind turbine structure. The drawback of the method in [25] is that the number of measurements must be at least as many as the number of parameters and inputs of interest, and at least as many displacement measurements as unknown inputs. This approach is therefore not suitable for the parametric output-only identification of the hysteretic system in the present benchmark challenge, where only a single response output is available.

The existing output-only identification strategies offer limited estimation accuracy for identification of nonlinearities in dynamic structures and limited attention is in particular offered for the estimation of mechanisms related to energy dissipation, which may be a dominant source of nonlinearity. The existing output-only techniques may therefore in particular fail for nonlinear systems which are excited by high-levels of amplitude. Systems with nonlinear damping mechanisms can be well represented by equivalent linear models [27], but may not be representative when the nonlinearities in the stiffness are severe and cause jump and bifurcation phenomena. A review of the diverse sources of nonlinear damping in practical systems is given in [27] as well as linearizaton techniques. Regardless of the philosophy of the identification method, the dynamic nonlinearities in hysteretic systems are difficult to identify, since it is not possible to directly measure the internal state variable which controls the hysteretic evolution. The parameter identification of the hysteretic system is herein treated by deriving an equivalent linear relaxation model, whose parameters depend on the magnitude of the excitation and frequency. Such an implicit model offers a system format that is observable and controllable, and simplifies the analysis. This leads to a computationally efficient identification methodology that extends the classical output-only covariance driven stochastic subspace identification [4] and its ability to estimate the model stiffness and damping at varying amplitudes of excitation.

The Bouc-Wen model $[28,29]$ is proposed for the synthetic generation of data for the identification challenge, which is used extensively in modeling of the hysteresis phenomenon in dynamically excited nonlinear structures [30, 31]. Various suitable methods are developed in structural dynamics as means for solving nonlinear stochastic problems [32-34], such as the Bouc-Wen model. These rely on linearization techniques and are commonly useful for approximate techniques in the first design stage. Therefore, an obvious method for solving the nonlinear identification challenge is to replace the governing set of nonlinear differential equations by an equivalent set of linear equations. The linearization turns out to be an advantage, which enables the output-only covariance driven stochastic subspace identification to be applied for the benchmark challenge. The starting point of the system identification is the ability to estimate the model parameters of single output.

The proposed method is therefore an approach suitable for a single-degree-of-freedom oscillator with viscoelastic properties. In the case of a Gaussian excitation, the response of a weakly nonlinear system can be 
assumed to approach a Gaussian distribution [34]. The identification procedure can therefore be appropriately based on the statistical properties which are necessary for the covariance driven identification methods. The novel method estimates the parameters of a dynamic model in the form of coupled ordinary differential equations, wherein the hysteresis phenomenon is represented by the Bouc-Wen model. The model parameters are related through derived expressions of the parameters of a linear relaxation damping model. To validate the proposed identification method a synthetic data set with unknown signal noise is made available from the source [5]. The effect of the equivalent linear model on the identification of the hysteretic system is discussed and the paper concludes on future steps in the direction of output-only system identification for hysteretic systems excited at high-level input.

\section{Linear system model of the hysteretic benchmark challenge}

The equation of motion describing the vibrations of a single-degree of freedom system can be expressed as

$$
m \ddot{x}(t)+c \dot{x}(t)+k x(t)+z(x, \dot{x})=u(t)
$$

where $m$ is the mass, $c$ is the viscous damping coefficient, $k$ is the stiffness, $f(t)$ is the external force, $x(t)$ is the displacement, while the derivatives $\dot{x}(t)$ and $\ddot{x}(t)$ represent velocity and acceleration, respectively. The restoring force $z(x, \dot{x})$ of a hysteretic system can be described by the mathematical Bouc-Wen model

$$
\dot{z}(x, \dot{x})=\alpha \dot{x}(t)-\beta\left(\gamma|\dot{x}(t)||\dot{z}(t)|^{\nu-1} z+\delta \dot{x}(t)|z(t)|^{\nu}\right),
$$

where $\alpha, \beta \gamma, \beta \delta$ and $\nu$ are parameters, controlling the shape and smoothness of the hysteresis loop. Details about the Bouc-Wen model can be found in [5], while its mechanical representation is shown in Figure 1(a). The theoretical development for response characteristics of nonlinear systems subjected to random excitation have not yet been reduced to generally applicable results. In principle an exact solution for the response of any dynamical system (linear or nonlinear) subjected to white Gaussian excitation can be obtained from the theory of the continuous Markov processes. The exact closed form solutions are only available for certain

(a)

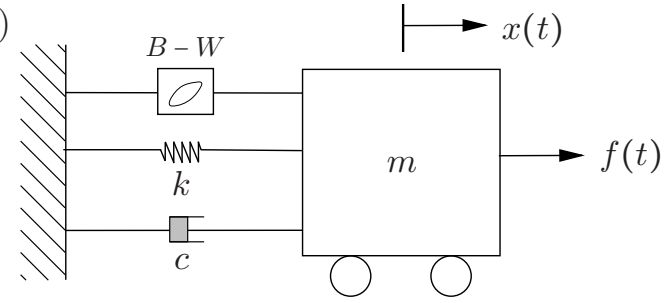

(b)

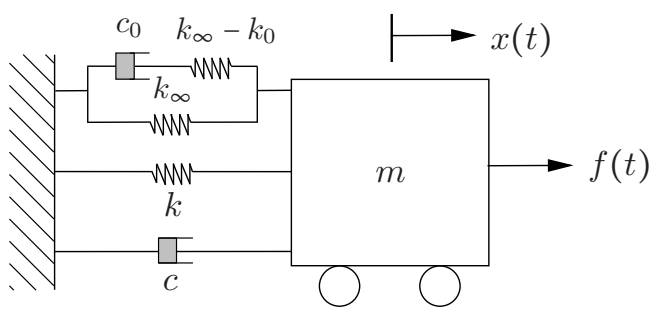

Figure 1: Mechanical representation of a viscous spring-mass oscillator with (a) a Bouc-Wen (B-W) restoring force and (b) a relaxation element with $c_{0}=\left(k_{\infty}-k_{0}\right) / \Omega$. 
special cases, and therefore solved by approximate methods. Linearization methods approximate the solution by replacing the governing set of nonlinear differential equations by a set of linear differential equations.

\subsection{Equivalent linear model}

The principle of harmonic averaging for hysteretic systems was originally demonstrated in [32]. The validity of the method is limited to systems with slowly varying amplitude and phase, and scenarios in which the response is narrow banded with small energy dissipation per cycle. The stochastic linearization method can cope with wide band response. Such a method obtains the model parameters of the equivalent system by minimizing the mean square of the difference between the nonlinear and the equivalent linear expressions. However, the involved evaluation of the expectations results in a stochastic linear model in which the standard deviation of the unmeasured internal state variable and its correlation coefficient appear [33, 34]. Thus, stochastic linearization is seemingly inappropriate for output-only system identification purposes. The method of stochastic linearization has proven useful in the design of earthquake resistance structures [33]. The response is, however, non-stationary and therefore not suitable for output-only identification by the stochastic subspace identification method, due to the underlying assumption of stationary output. For identification from structural response during earthquakes the method described in [35] is therefore more appropriate. Furthermore, perturbation methods are avoided in the present context because of their increased algebraic complexity and limited accuracy up to first order. Therefore, an equivalent linear model is described for the present hysteretic system, which can be calibrated by a harmonic averaging principle [36]. The first step is to replace the nonlinear model by introduction of an equivalent linear model. A wide range of possible candidate models exist, but the introduction of a temporal model memory is conveniently represented by the first order differential equation [37]

$$
\dot{z}(t)+\Omega z(t)=\Omega k_{0} x(t)+k_{\infty} \dot{x}(t),
$$

where $\Omega, k_{0}$ and $k_{\infty}$ in the following are related to the Bouc-Wen parameters $\alpha, \beta \gamma$ and $\beta \delta$ in (2) by harmonic averaging over an assumed vibration period. The format of (3) can be recognized as an nonviscous damping model with relaxation properties, often also referred to as a viscoelastic material model. The phenomenological representation of the equivalent linear model with mechanical elements is shown in Figure 1(b). The model was originally introduced by Zener [38] and encompasses a combination of a Kelvin-Voigt model with a spring $k_{0}$ and dashpot $c_{0}$ in parallel, and with an additional spring $k_{\infty}-k_{0}$ in series with the dashpot $c_{0}$. Hereby, $k_{0}$ represent the low-frequency stiffness of the relaxation model, while $k_{\infty}$ appears as the model stiffness in the high-frequency range. In the intermediate range at the corner frequency $\Omega$, the magnitude of the energy dissipation is determined by the stiffness change $k_{\infty}-k_{0}$. Because the low-frequency stiffness $k_{0}$ can be directly combined with the structural stiffness $k$, the relaxation model 
in (3) may be readily reduced to the two-parameter model associated with vanishing $k_{0}$, which is further emphasized in the following derivation.

The linearization technique of the Bouc-Wen model is demonstrated for the particular case of $\nu=1$, reducing the nonlinear restoring force in (2) to

$$
\dot{z}(x, \dot{x})+\beta \gamma|\dot{x}(t)| z=(\alpha-\beta \delta|z(t)|) \dot{x}(t) .
$$

Due to the inherent relaxation properties of both (3) and (4), the relation between the model parameters are determined in the two limits associated with slow and fast motion, or low and high vibration frequency, relative to the corner frequency $\Omega$. For a broad-banded excitation of a lightly-damped structure, it can be assumed that the structural response is fairly narrow-banded, whereby the response can be approximated between two up-crossings by the harmonic expressions

$$
x(t)=A \cos (\omega t) \quad \text { and } \quad \dot{x}(t)=-A \omega \sin (\omega t),
$$

with amplitude $A$ and angular frequency $\omega$. This harmonic motion is in the following assumed when obtaining the system parameters of the equivalent linear relaxation model in (3).

\subsection{Low-frequency equivalence}

Initially, the Bouc-Wen model is represented in the low-frequency limit, associated with slow motion. In the low-frequency range the time differentiated terms $\dot{z}(t)$ and $\dot{z}(x, \dot{x})$ on the left hand sides of (3) and (4) respectively, are assumed to vanish. Thus, the low-frequency representation of the Bouc-Wen model (4) can be written as

$$
\beta \gamma|\dot{x}(t)| z(t)=(\alpha-\beta \delta|z(t)|) \dot{x}(t)
$$

while the corresponding low-frequency relaxation model is

$$
\Omega z(t)=\Omega k_{0} x(t)+k_{\infty} \dot{x}(t)
$$

where the first term represents the low-frequency stiffness, while the second viscous term represents damping. The stiffness and viscous damping contributions are identified separately. The low-frequency component of the stiffness is assumed to be in-phase with the displacement and can therefore be approximated from (7) as

$$
z(t)=k_{0} x(t)=k_{0} A \cos (\omega t) .
$$

This expression for $z(t)$ is now inserted into the low-frequency Bouc-Wen model in (6). In this case the low-frequency stiffness $k_{0}$ is determined by multiplication with $\cos (\omega t)$ and integrating the relation over a full vibration period,

$$
\int_{0}^{2 \pi / \omega}\left[k_{0} A^{2} \omega \beta \gamma|\sin (\omega t)| \cos ^{2}(\omega t)+\left(\alpha-\beta \delta\left|k_{0}\right| A|\cos (\omega t)|\right) A \omega \sin (\omega t) \cos (\omega t)\right] d t=0 .
$$


Evaluation of the integral reveals that the low-frequency linear stiffness component vanishes such that

$$
k_{0}=0,
$$

which demonstrates that the low-frequency stiffness is contained in the structural stiffness component $k$.

The viscous part in the linear low-frequency model is assumed to be in-phase with velocity, whereby it follows from (7) as

$$
z(t)=\frac{k_{\infty}}{\Omega} \dot{x}(t)=-\frac{k_{\infty}}{\Omega} A \omega \sin (\omega t),
$$

in which $\dot{x}(t)$ is substituted from (5) and $k_{0}=0$. This assumed expression for $z(t)$ is substituted into the low-frequency form of the Bouc-Wen model in (6), which is then multiplied by $\sin (\omega t)$ and integrated over a single vibration period,

$$
\int_{0}^{2 \pi / \omega}\left[-\frac{k_{\infty}}{\Omega} \beta \gamma A^{2} \omega^{2}|\sin (\omega t)| \sin ^{2}(\omega t)+\left(\alpha-\beta \delta\left|\frac{k_{\infty}}{\Omega}\right| A \omega|\sin (\omega t)|\right) A \omega \sin ^{2}(\omega t)\right] d t=0 .
$$

Evaluation of the integral then gives an expression for the stiffness to frequency ratio

$$
\frac{k_{\infty}}{\Omega}=\frac{3 \pi}{4} \frac{\alpha}{2 A \omega(\beta \gamma+\beta \delta)} .
$$

This ratio represents the damping parameter of the linear relaxation and therefore $\frac{k_{\infty}}{\Omega}>0$. Because of the nonlinearities in the original model, the equivalent linear parameter in (13) becomes amplitude dependent, and for increasing $A \omega$, the linear parameter $\frac{k_{\infty}}{\Omega}$ will apparently decrease.

\subsection{High-frequency equivalence}

In the high-frequency limit associated with fast motion, the time derivative term on the left hand side of the Bouc-Wen model in (4) becomes dominant and the model is approximated as

$$
\dot{z}(t)=(\alpha-\beta \delta|z(t)|) \dot{x}(t) .
$$

The corresponding linear relaxation model in (3) can similarly be reduced to

$$
\dot{z}(t)=\Omega k_{0} x(t)+k_{\infty} \dot{x}(t)
$$

Again the viscous and stiffness contributions are identified separately. First, the viscous part of the highfrequency relaxation model in (15) is approximated by

$$
\dot{z}(t)=\Omega k_{0} x(t) .
$$

As $k_{0}=0$ this contribution vanishes and therefore the present linearization only leaves a high-frequency stiffness component. This stiffness contribution can be obtained from (15) as

$$
\dot{z}(t)=k_{\infty} \dot{x}(t)=-k_{\infty} A \omega \sin (\omega t) .
$$


When inserting this simplified harmonic representation into the high-frequency Bouc-Wen model (14), the stiffness $k_{\infty}$ can be isolated by multiplying the relation with $\sin (\omega t)$ and integrating it over an assumed vibration period,

$$
\int_{0}^{2 \pi / \omega}\left[-k_{\infty} A \omega \sin ^{2}(\omega t)+\left(\alpha-\beta \delta\left|k_{\infty}\right| A \cos (\omega t) \mid\right) A \omega \sin ^{2}(\omega t)\right] d t=0 .
$$

Evaluation of the integral leads to

$$
k_{\infty}=\frac{3 \pi}{4} \frac{\alpha}{A \beta \delta+\frac{3 \pi}{4}} .
$$

It is important to note that energy dissipation by the Zener type relaxation model in (3) requires that $k_{\infty}-k_{0}$ is positive, which in the present case corresponds to $k_{\infty}>0$. It follows from (19) that for small vibration amplitudes $A$, the equivalent stiffness $k_{\infty}$ approaches the initial stiffness component $\alpha$ in the Bouc-Wen model, while for finite amplitude levels the stiffness is either reduced or increased, depending on the sign of $\beta \delta$. The corner frequency $\Omega$ in the relaxation model (3) is now obtained by dividing the expression in (19) with that in (13). This gives

$$
\Omega=2 A \omega \frac{\beta \gamma+\beta \delta}{A \beta \delta+\frac{3 \pi}{4}}
$$

which is valid for $\Omega>0$. Thereby, a relationship is established between the parameters of the linear model and the Bouc-Wen model parameters, based on harmonic averaging in the low- and high-frequency limits. The underlying assumption is that if the two frequency limits are well calibrated, the equivalence between the two models is also reasonable in the intermediate frequency range. When the parameters of the linear relaxation model are determined by the system identification procedure, the Bouc-Wen model parameters can subsequently be found by the relations in (19) and (13).

\subsection{Numerical example of the equivalent linear system}

In the following the effect of the obtained equivalent model is illustrated for a set of parameter values of the hysteretic system. The parameters of the linear system have been set to $m=2 \mathrm{~kg}, k=50000 \mathrm{~N} / \mathrm{m}$, $c=10 \mathrm{Ns} / \mathrm{m}$ and $\alpha=50000 \mathrm{~N} / \mathrm{m}$, whereby the natural frequency of the linear system is $\omega=223.61 \mathrm{rad} / \mathrm{s}$. The Bouc-Wen model parameters in the simulation have been chosen to be $\beta \gamma=800 \mathrm{~m}^{-1}$ and $\beta \delta=-1100 \mathrm{~m}^{-1}$. An explicit time integration scheme using a fixed-step fourth order Runge-Kutta solver has been used to simulate the response. The system has been excited by a random process, obtained from a normal distribution of random numbers generated in MATLAB. The excitation amplitude has been scaled to obtain a root-meansquare amplitude of $30 \mathrm{~N}$. The displacement time history has been sampled with $375 \mathrm{~Hz}$ and $N=2^{20}$ number of points. The corresponding time step of $\Delta t=0.0027 \mathrm{~s}$ is greater than one-tenth of the natural period in the system, and thereby the Runge-Kutta solver is expected to have a reasonable computational speed and accuracy. The simulation length is equivalent to 99512 vibration periods of the linear vibration mode. Figure 
(a)

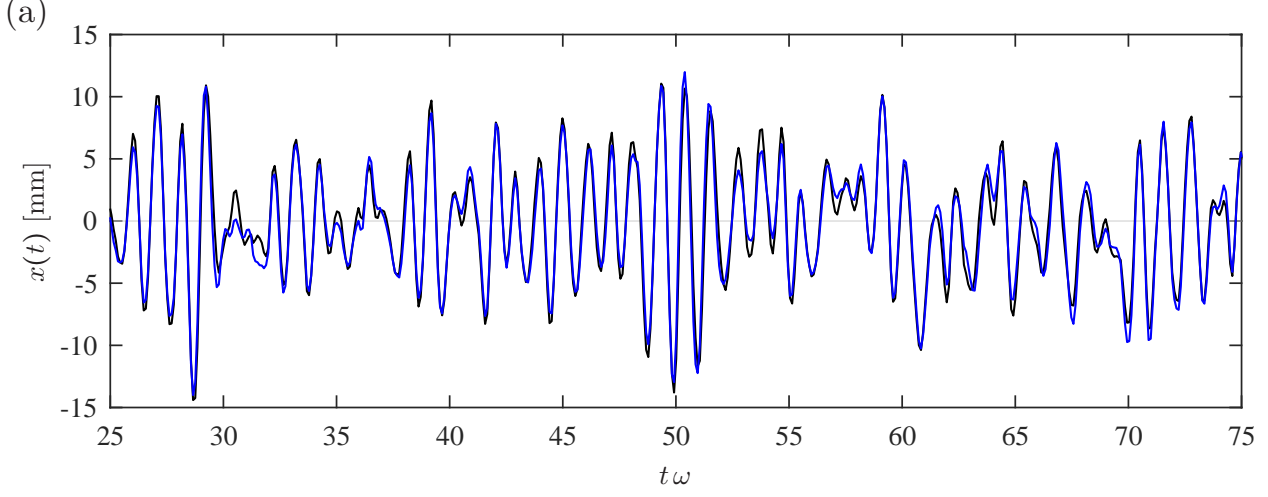

(b)

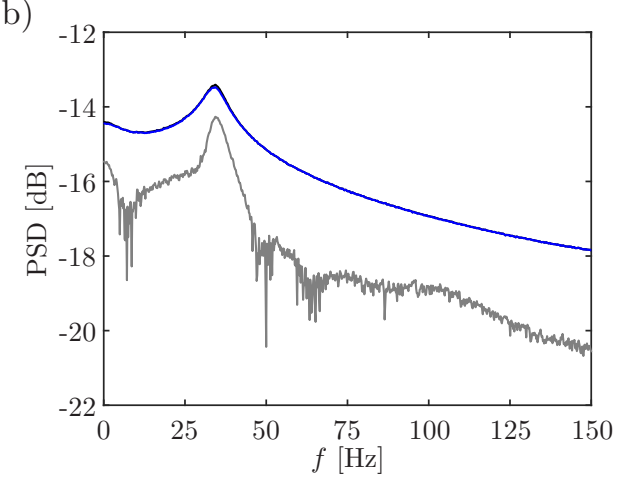

(c)

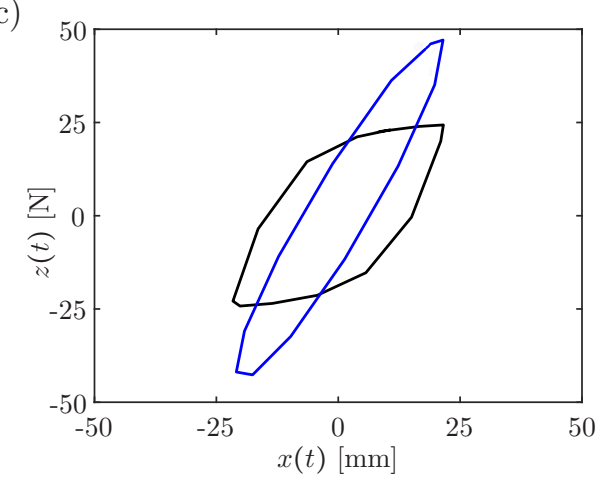

Figure 2: The synthetic random response of the equivalent linear model (-) superimposed on the response of the hysteretic system (-). (a) The displacement time history, (b) the power spectral density (PSD) with the difference between the models in $(-)$ and (c) the restoring force in the system.

2 shows the response computed for initial conditions set to zero, which shows a non-degenerated hysteresis loop.

The system model parameters of the equivalent linear system are $k_{\infty}=44774 \mathrm{~N} / \mathrm{m}, \Omega=80.76 \mathrm{rad} / \mathrm{s}$ and $k_{0}=0 \mathrm{~N} / \mathrm{m}$. These are obtained by the expressions in (19) and (20), where the natural period of the linear system has been chosen as $\omega=223.61 \mathrm{rad} / \mathrm{s}$, while the amplitude $A=2.1 \mathrm{~mm}$ represents the standard deviation of the displacement response of the hysteretic system.

The displacement and power spectral density (PSD) of the equivalent linear system, shown in Figure 2(a)(b), demonstrate a good agreement between the hysteretic and equivalent linear system. The PSD has been computed using Welch's method of spectral estimation, applying a Hanning window for the averaging with an overlap of $50 \%$. The hystersis loop of the equivalent linear system in Figure 2(c) is more smooth and narrow, while the enclosed areas are almost identical, indicating a reasonable representation of the energy dissipation in the system. 


\section{Extended covariance driven parameter identification}

The hysteretic benchmark problem is considered as a state estimation problem for the discrete-time system. It is expressed in the classic form

$$
\begin{aligned}
& \dot{\mathbf{x}}(t)=\mathbf{A} \mathbf{x}(t)+\mathbf{B} \mathbf{u}(t)+\mathbf{E} \mathbf{e}(\mathbf{x}(t), \mathbf{f}(t)) \\
& \mathbf{y}(t)=\mathbf{C} \mathbf{x}(t)+\mathbf{D} \mathbf{u}(t)+\mathbf{F} \mathbf{f}(\mathbf{x}(t), \mathbf{f}(t))
\end{aligned}
$$

where $\mathbf{x}$ is the $n \times 1$ state vector, $\mathbf{y}$ is the $m \times 1$ output vector, $\mathbf{u}$ is the $r \times 1$ output vector, $\mathbf{A}$ is the $n \times n$ state matrix, $\mathbf{B}$ is the $n \times r$ input matrix, $\mathbf{C}$ is the $m \times r$ output matrix and $\mathbf{D}$ is the $m \times r$ freedthrough matrix. For nonlinear systems $\mathbf{E}, \mathbf{F}, \mathbf{f}$ and $\mathbf{e}$ are established, which include the combinations of the state and input variables. Thus, their individual size depends on the specific system to be analyzed. The state estimation problem corresponds to the Kalman filtering problem. However, in the present case the system parameters in the matrices are sought, instead of the time history of the state variables. The so-called stochastic subspace identification methods are formulated for this specific purpose. The key point of an output-only stochastic subspace method relies on the fact that the system Markov parameters can be obtained in observable and controllable systems by the impulse response of the system, which is represented in a Toeplitz matrix structure. The nonlinear hysteretic system from (4) can be written in state space format as

$$
\begin{aligned}
{\left[\begin{array}{l}
\dot{x}(t) \\
\ddot{x}(t) \\
\dot{z}(t)
\end{array}\right] } & =\left[\begin{array}{ccc}
0 & 1 & 0 \\
-k / m & -c / m & -1 / m \\
0 & \alpha & 0
\end{array}\right]\left[\begin{array}{c}
x(t) \\
\dot{x}(t) \\
z
\end{array}\right]+\left[\begin{array}{c}
0 \\
1 / m \\
0
\end{array}\right] f(t)+\left[\begin{array}{cc}
0 & 0 \\
0 & 0 \\
-\beta \gamma & -\beta \delta
\end{array}\right]\left[\begin{array}{l}
|\dot{x}(t)| z(t) \\
\dot{x}(t)|z(t)|
\end{array}\right] \\
y(t) & =\left[\begin{array}{lll}
1 & 0 & 0
\end{array}\right]\left[\begin{array}{lll}
x(t) & \dot{y}(t) & z(t)
\end{array}\right]^{T},
\end{aligned}
$$

where ${ }^{T}$ denotes the transpose. This shows that the hysteretic system is in fact not observable and controllable, due to the rank deficiency of the observability $\mathcal{O}=\left[\begin{array}{l}\mathbf{C C A}\end{array}\right]^{T}$ and controllability $\mathcal{C}=\left[\begin{array}{ll}\mathbf{B} & \mathbf{A B}\end{array}\right]$ of the system. Therefore, the system cannot be estimated directly from the linear subspace identification method. The linearized relaxation system in (3) can also be formulated in a state-space setting,

$$
\begin{aligned}
{\left[\begin{array}{l}
\dot{x}(t) \\
\ddot{x}(t) \\
\dot{z}(t)
\end{array}\right] } & =\left[\begin{array}{ccc}
0 & 1 & 0 \\
-k / m & -c / m & -1 / m \\
0 & k_{\infty} & -\Omega
\end{array}\right]\left[\begin{array}{c}
x(t) \\
\dot{x}(t) \\
z
\end{array}\right]+\left[\begin{array}{c}
0 \\
1 / m \\
0
\end{array}\right] f(t) \\
y(t) & =\left[\begin{array}{lll}
1 & 0 & 0
\end{array}\right]\left[\begin{array}{lll}
x(t) & \dot{y}(t) & z(t)
\end{array}\right]^{T},
\end{aligned}
$$

where $\Omega k_{0}=0$ has been demonstrated in (10). The linear time-invariant system in (25)-(26) is identifiable, since the observability and controllability matrices have the desired rank. The proposed identification procedure therefore relies on the linearized model in (25)-(26) and it consists of the steps explained next. 


\subsection{Correlation functions of stationary processes}

The unbiased auto-correlation function estimate from the random response $x(t)$ of the oscillator is obtained by the discrete representation

$$
\hat{R}_{x x}(m)=\frac{1}{N-m} \sum_{n=0}^{N-1-m} x(n) x(n+m),
$$

for $n=m=0,1,2, \ldots, N-1$, where $x(n)$ is a discrete representation with time step $n$ of the corresponding stochastic realizations of $x(t)$. The discrete representation in (27) is related to the continuous representation by the time $t=n \Delta t$, the time lag $\tau=m \Delta t$ and the total time $T=(N-1) \Delta t$, and it is valid for positive time lags $\tau>0$.

The output-only covariance driven stochastic subspace identification is performed by the original formulation in [4]. The observability and controlability matrices are estimated by a singular value decomposition of the block-Toeplitz matrix which contains the correlations of the output. Thus, the discrete-time dynamic matrix A can be established from the observability, controlability and the shifted block-Toepelitz matrix. The discrete time poles are subsequently found by decomposing the dynamic system matrix into the correspoding eigenvectors and eigenvalues at a number of model orders. A complex pole $\mu$ associated with discrete time is related to the corresponding continuous time pole $\lambda$ via the relation

$$
\lambda=\frac{\ln (\mu)}{\Delta t},
$$

where $\Delta t$ is the chosen sampling time increment. The continuous poles can then be related to the natural frequency and the damping of the targeted vibration mode of the system by

$$
\lambda=-\omega \zeta \pm i \omega_{D}
$$

where $i=\sqrt{-1}$ is the imaginary unit, $\omega_{D}=\omega \sqrt{1-\zeta^{2}}$ is the damped natural angular frequency, $\omega$ is the undamped natural angular frequency and $\zeta$ is the modal damping ratio.

\subsection{Identification of the stabilized poles}

The identification method relies on the estimation of the complex poles of the vibratory system. These are therefore an important contribution to a successful identification of the system. The truncation of singular values may be challenging, in particular for noisy data records. Thus, the poles are selected by their ability to fulfill the so-called stabilization criteria at model orders $j$ and $h$. The stabilization criteria have been introduced in numerous automated versions of the subspace identification methods [39-41]. These typically identify a cluster of stabilized poles for specific thresholds of the relative difference in either the natural angular frequency $\omega$ or the damping ratio $\zeta$, estimated at model order $j$ relative to model order $h$ by the expressions

$$
d \omega_{h, j}=\frac{\left|\omega_{h}-\omega_{j}\right|}{\max \left(\omega_{h}, \omega_{j}\right)} \text { and } d \zeta_{h, j}=\frac{\left|\zeta_{h}-\zeta_{j}\right|}{\max \left(\zeta_{h}, \zeta_{j}\right)} .
$$


A cluster of stabilized poles is identified by the differences $d \omega_{h, h+1}$ and $d \zeta_{h, h+1}$ between two adjacent model orders $h$ and $j=h+1$ for a predefined threshold value of $d \omega_{h, j}$ and $d \zeta_{h, j}$.

For the linear system in (25) considered in the present identification procedure, the formed clusters will contain the stabilized pole $\lambda_{i}$ and its complex conjugate $\lambda_{i}^{*}$. These two poles are related to the dynamic modes and they determine the characteristics of the oscillatory part of the system. However, because of the memory effect of the relaxation model in (3), an additional real-valued root can be found for the system recast into the state space format in (25), which represents the time delay introduced by the relaxation process of the Zener model. This auxiliary third pole is therefore associated with an overdamped vibration mode without an oscillatory character $[42,43]$. Such pole is determined by the additional stabilization criteria

$$
d r_{h, j}=\frac{\left|\lambda_{h}-\lambda_{j}\right|}{\max \left(\lambda_{h}, \lambda_{j}\right)} \quad \text { where } \operatorname{Im}(\lambda)=0 .
$$

This is found to identify the additional stabilized real-valued pole for a certain threshold value $d r_{h, j}$, which is denoted $\lambda_{r}$. The cluster of estimated real-valued poles can stabilize with respect to the frequency $d \omega_{i, j}$. However, poles without any imaginary part will not have any variation in the damping ratio for increasing model order, and thus they will not fulfill the stabilization criteria $d \zeta_{h, j}$ associated with damping.

\subsection{Identification from the characteristic polynomial structure}

In the following it is presented how the two identified complex conjugated poles and the additional third real pole of the estimated dynamic state matrix enable estimation of the linear stiffness $k$, and the constants $k_{\infty}$ and $\Omega$ of the equivalent linear relaxation model. The characteristic polynomial obtained from the dynamic matrix in $(25)$ is

$$
-\lambda_{i}^{3} m+(-\Omega m-c) \lambda_{i}^{2}+\left(-\Omega c-k-k_{\infty}\right) \lambda_{i}-k \Omega=0 .
$$

When the three stabilized poles $\lambda_{i}$ (where $i=1,2$ and 3) are inserted, the solution to (32) gives an expression for $k, k_{\infty}$ and $\Omega$. These are

$$
\begin{gathered}
k=\frac{m^{2} \lambda_{1} \lambda_{2} \lambda_{3}}{\left(\lambda_{1}+\lambda_{2}+\lambda_{3}\right) m+c}, \\
k_{\infty}=-\frac{\left(m \lambda_{1}{ }^{2}+c \lambda_{1}+k\right)\left(m \lambda_{2}{ }^{2}+c \lambda_{2}+k\right)}{-m \lambda_{1} \lambda_{2}+k}
\end{gathered}
$$

and

$$
\Omega=\lambda_{1}\left(1+\frac{k_{\infty}}{m \lambda_{1}^{2}+c \lambda_{1}+k}\right) .
$$

These three expressions establish a relation between the three estimated poles $\lambda_{i}$ and the linear parameters $k, k_{\infty}$ and $\Omega$. The identification procedure requires an assumed mass $m$ of the oscillator, and an initially estimation of the viscous damping coefficient $c$, such that the expressions (33)-(35) can be used for estimation. 


\subsection{Identification of the Bouc-Wen model parameters}

The characteristic polynomial related to the dynamic matrix in (23) of the Bouc-Wen model will have the three roots,

$$
\lambda=\left\{\begin{array}{l}
0 . \\
\frac{-c}{2 m} \pm \frac{\sqrt{c^{2}-4 m(\alpha+k)}}{2 m} .
\end{array}\right.
$$

This shows that the system has two complex conjugated poles and a strictly zero pole. If the constant $\Omega<<1$ in the equivalent linear model, the poles in (36) can be related to the poles in (32). Thereby, the parameter $\alpha$ of the Bouc-Wen model can be related to the imaginary part of the non-zero pole in (36) by

$$
\alpha=\frac{c^{2}}{4 m} \pm \operatorname{Im}\left(\lambda_{i}\right)^{2} m-k,
$$

where $\lambda_{i}$ must have a non-zero imaginary part, which requires $c^{2}<4 m(\alpha+k)$. Once $\alpha$ is estimated, the remaining Bouc-Wen model parameters are found by rearranging the expressions in (19)-(20) such that

$$
\beta \delta=\frac{3 \pi}{4 A}\left(\frac{\alpha}{k_{\infty}}-1\right) \text { and } \beta \gamma=\frac{3 \pi}{4 A}\left(1+\frac{\Omega \alpha}{2 \omega k_{\infty}}-\frac{\alpha}{k_{\infty}}\right),
$$

where the amplitude $A$ can be chosen as the standard deviation $\sigma_{x}$ of the measured response time history $x(t)$, such that $A=\sigma_{x}$. The natural angular frequency of the oscillator can be estimated by any of the two complex conjugated poles, as $\omega=\left|\lambda_{i}\right|$. It should be noted that the modified stochastic subspace identification method can operate for both stochastic realizations of the accelerations $\ddot{x}(t)$, the velocities $\dot{x}(t)$ and displacements $x(t)$. This concludes the presentation of the identification method for the hysteretic benchmark problem. The method will be demonstrated and validated in the following, initially for synthetic data of the random response generated for the mechanical model illustrated in Figure 1(a).

\section{Identification results}

The proposed identification procedure is firstly demonstrated by the provided data set described in detail in [5], which represents the response of the hysteretic system simulated by the Bouc-Wen model in (1) and (2). The dataset containing the response due to sine-sweep excitation will not be the target of the identification. In the final part of this section, the estimation procedure will be discussed for low, middle and high-level input magnitude of the random excitation. The input has not been used at any point during the identification, but it has inevitably been used to simulate the response of the estimated system. The estimated model parameters have been evaluated by comparing the estimated and the simulated response. The benchmark problem dataset is generated by applying a random phase multi-sine excitation with a rootmean square excitation amplitude of $50 \mathrm{~N}$. The excitation is band limited to excite frequencies in the range 
(a)

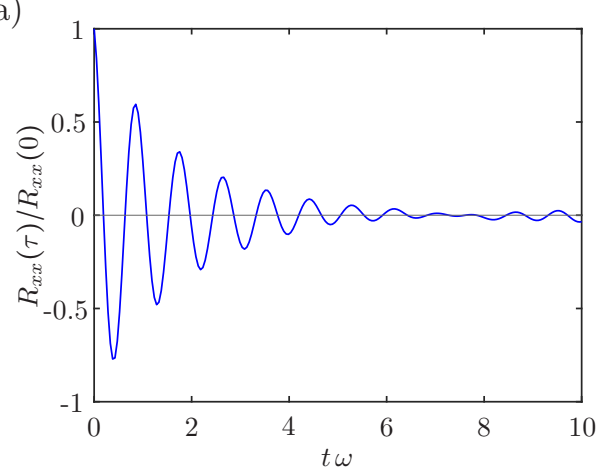

(b)

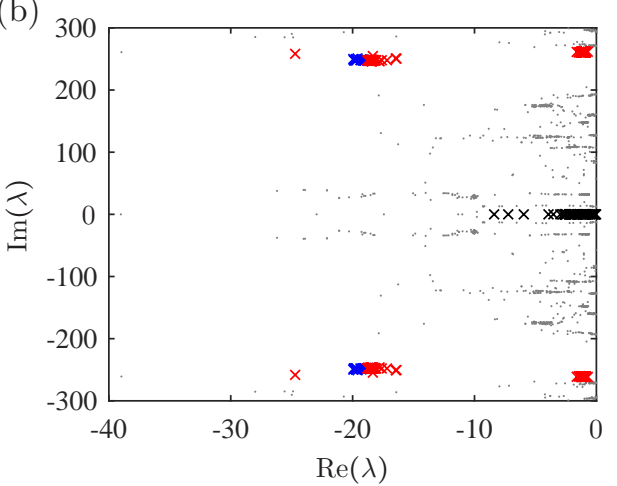

Figure 3: (a) The unbiased correlation function estimate. (b) The identified continuous time poles (.) stabilized in frequency and damping $(\times)$, stabilized in frequency $(x)$ and stabilized in real value $(x)$.

$5-150 \mathrm{~Hz}$. The steady state response is sampled with $750 \mathrm{~Hz}$ and contains 8192 samples for a hysteretic system with model parameters equivalent to the system in Section 2.4 as later shown in Figure 5 . The power spectral density of the data indicates that the system has a natural frequency at approximately $40 \mathrm{~Hz}$.

To avoid issues in the identification due to over-sampling the output data is down-sampled. This has been achieved by applying the function decimate in MATLAB, with a 30th order FIR filter, which can handle possible edge effects of the down-sampling. After the down-sampling the kurtosis is 2.59 and the skewness is -0.02 , indicating that the dataset can be regarded as a zero-mean stationary process.

The unbiased auto-correlation function estimate of the output data is shown in Figure 3(a), where the first ten periods exhibit an exponential decay. The size of the block-Toeplitz matrix has been established for $\tau$ corresponding to 10 oscillation periods. The two stabilization criteria in (30) have both been set to 0.05 . The criteria in (31) for the real valued pole has been set to 1.5, which is substantially higher than the threshold value of 0.05 for (30), as the threshold limit is related to the real value only. Figure 3 (b) shows the continuous time poles obtained by the SSI method and the model parameters of the equivalent linear system are then obtained by evaluating these identified poles.

The identification shows that the poles form five clusters, see Figure 3(b), where one of these is located on the real axis. The cluster on the real axis is related to the non-oscillatory part of the system and has a mean value of -1 . The two clusters with positive imaginary parts are formed at two distinct real values, with corresponding complex conjugated clusters. Figure 4(a) shows the stabilization of the identified frequency and indicates that the pole with the lowest estimated frequency stabilizes in both damping and frequency at low model orders. At a higher model order this pole splits with two distinct frequencies at approximately $39.5 \mathrm{~Hz}$ and $41.5 \mathrm{~Hz}$. The damping ratios identified are given in Figure 4(b), which indicates that both clusters stabilize in frequency for increasing model orders, whereas only the pole with low real values stabilizes in damping. The damping ratios of the two clusters stabilized in frequency are approximately 0.004 and 0.074 . 
(a)

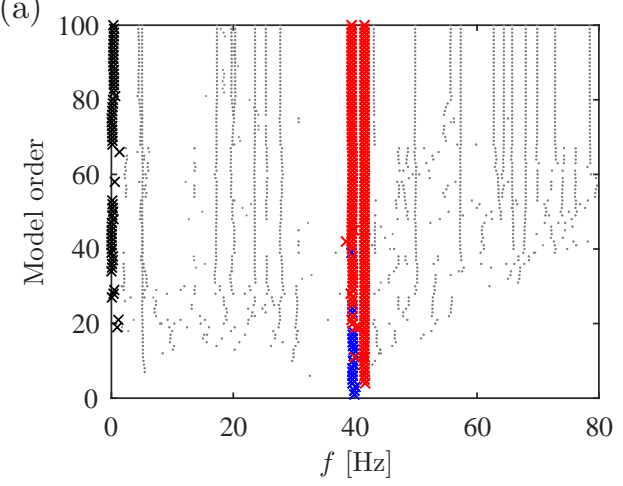

(b)

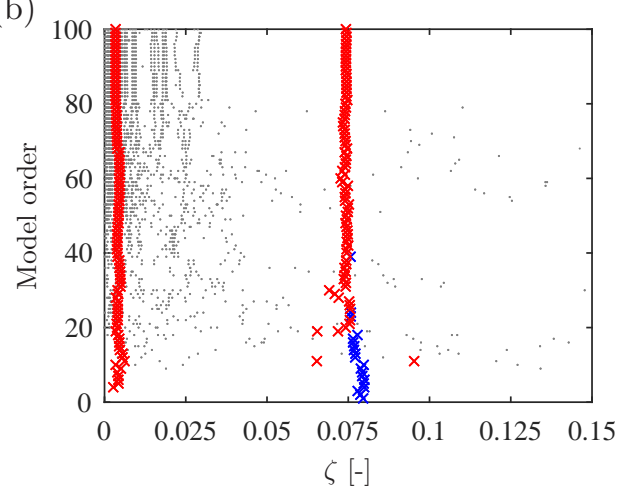

Figure 4: (a) Identified frequencies and (b) damping ratios for variations in model order. Poles (.) stabilized in frequency and damping $(x)$, stabilized in frequency $(x)$ and stabilized in real value $(x)$.

The mass of the system is assumed to be equal to $2 \mathrm{~kg}$. With this assumed mass, the linear system has been estimated from the two complex conjugated poles which stabilize in both damping and frequency at a model order of 8 . The selected poles have the value $\lambda=-19.9414 \pm 249.7904 i$, which result in a damping coefficient of $79.77 \mathrm{Ns} / \mathrm{m}$ and a stiffness of $125590 \mathrm{~N} / \mathrm{m}$. It should be noted that the damping coefficient and the stiffness are not the parameters of the purely linear system, but contain a contribution from the combined linear and nonlinear system in the dataset. The time domain and frequency domain response of the identified combined linear system is illustrated in Figure 5. It initially shows that the identification of the linear system in the excited band predicts the resonance peak. However, at frequencies below and above the resonance the prediction of the estimated linear system deteriorates. As mentioned earlier the response has been generated with a fixed-step Runge-Kutta time integration scheme, with an excitation input from the dataset of the benchmark problem. It is hereby demonstrated that a linear system with an internal variable can be estimated by the proposed identification approach.

The identification of the equivalent linear system is expected to improve the accuracy of the predicted response. The model parameters of the equivalent linear system are determined by the expressions in (33)-(35). Thus, an initial guess of the damping coefficient $c$ is necessary. For this dataset a guess has been based on the cluster of poles found with a low real value, with a mean of $\operatorname{Re}(\hat{\lambda})=-1.6$. The damping coefficient is thereby $\hat{c}=-2 \operatorname{Re}(\lambda) m=6.40 \mathrm{Ns} / \mathrm{m}$. This pole is selected, because it is likely that the low damping ratio corresponding to such pole, is related to the constant damping coefficient of the linear system. An estimate of the equivalent linear model parameters have been obtained by (33)-(35), where the two complex conjugated poles are $\hat{\lambda}_{1,2}=-19.9414 \pm 249.7904 i$, selected at a model order equal to 8 as previously mentioned. The third pole is $\hat{\lambda}_{3}=-1$, representing the cluster of poles related to the non-oscillatory part of the system. The response of the identified relaxation damping model is illustrated in Figure 5, which demonstrates that the identification of the equivalent model parameters improves the estimation of the 
(a)

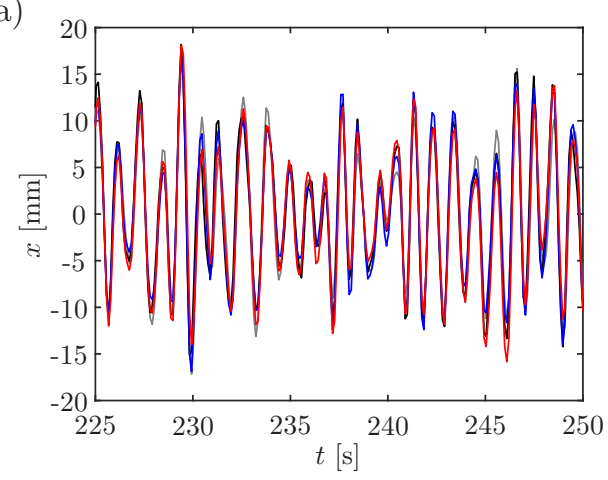

(b)

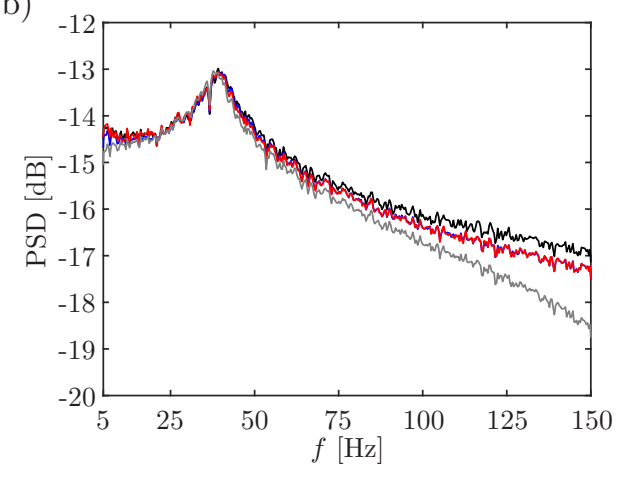

Figure 5: The estimated response superimposed on observed data. (a) Time history and (b) power spectral density (PSD). (-) Observed data, (-) estimated hysteretic system, (-) estimated equivalent linear system and (-) estimated linear system.

systems response. The model parameters have been estimated to $\hat{k}=3334.40 \mathrm{~N} / \mathrm{m}, \hat{k}_{\infty}=122150.00 \mathrm{~N} / \mathrm{m}$ and $\hat{\Omega}=37.68 \mathrm{rad} / \mathrm{s}$.

The final part of the identification procedure consists of estimating the Bouc-Wen model parameters from the identified parameters of the equivalent linear system. The hysteretic system adds linear stiffness by the parameter $\alpha$. The additional stiffness is estimated by (37). The imaginary part is obtained from the two complex conjugated poles: $\operatorname{Im}\left(\hat{\lambda}_{1,2}\right)= \pm 249.7904$. The remaining two parameters of the hysteretic system are obtained from the relations given in (38), where the standard deviation of the response time history is chosen as the vibration amplitude $\hat{A}=5.34 \mathrm{~mm}$, while the angular frequency of the system is $\hat{\omega}=\left|\hat{\lambda}_{1}\right|=250.59 \mathrm{rad} / \mathrm{s}$. The response of the estimated hysteretic system has been simulated with the properties given by the identified parameters. The hysteretic system parameters are thereby identified and the full system response is illustrated in Figure 5.

The estimated response can be evaluated by the root-mean-square deviation, obtained as

$$
\epsilon=\sqrt{\frac{1}{N} \sum_{n=0}^{N}(\hat{x}(n)-x(n))^{2}}
$$

for $n=0,1,2, \ldots, N-1$, where $N$ is the total number of points. Furthermore, $\hat{x}(n)$ is the estimated response, while $x(n)$ is the exact reponse given in the benchmark dataset at the discrete time step $n$. This error gives an indication of the average difference between the two time series. For the identified hysteretic systems, the equivalent linear system and the linear system the error is $2.55 \%, 2.20 \%$ and $3.31 \%$, respectively. The given values are normalized by dividing the expression in (39) with the range $\max (x(n))-\min (x(n))$, where $N=4096$. Furthermore, the error has been computed for the full time series, where the transient response is also included. The error decreases once the proposed identification method is used to estimate the model parameters. However, the average error of the identified equivalent linear model is slightly lower than the error of the hysteretic system. This indicates that the method identifies the parameters of the 
equivalent linear model more robustly than the hysteretic system. The improvements of the error offered by the identification method considering the hysteretic system parameters is relatively low. However, the method offers a valuable insight into the model parameters and their magnitude, which is of particular significance for high-levels of input excitation, as demonstrated in the following section.

The approach proposed is not expected to be error-free due to a number of reasons. The response is of finite duration and therefore some variance error in the unbiased auto-correlation function estimator in (27) will be introduced, even for a linear system. Secondly, the benchmark data has been down-sampled as described previously in this section. Thus, the reconstructed and the exact response are not expected to be identical. The identification procedure for hysteretic systems has been applied to a dataset which contains response of a system randomly excited with an amplitude that triggers the non-linear hysteresis state. This shows the applicability of the method for systems in which hysteretic effects can be observed. The dynamics of the hysteretic system will be affected by the amplitude of the excitation force, therefore the capabilities of the method at lower and higher excitation amplitudes are discussed in the next section.

\section{Identification at low- and high-level excitation amplitude}

The capabilities of the identification procedure are validated by comparing the exact response of the BoucWen system at a variation of excitation amplitudes, with the reconstructed response obtained from the proposed identification procedure in Section 3. The response is simulated as described in Section 2.4, where the excitation is as provided in [5]. The variation of the estimated model parameters, $\hat{\omega}, \hat{\zeta}, \hat{c}, \hat{k}, \hat{k}_{\infty}$ and $\hat{\Omega}$, with respect to the excitation level is initially discussed and shown in Figure 6-7. The excitation amplitude denoted by $|F|$ is computed by the standard deviation of the time series, and it ranges from 10 to $90 \mathrm{~N}$.

Figure 6 (a) shows a decreasing natural frequency with an increasing excitation amplitude, whereas the
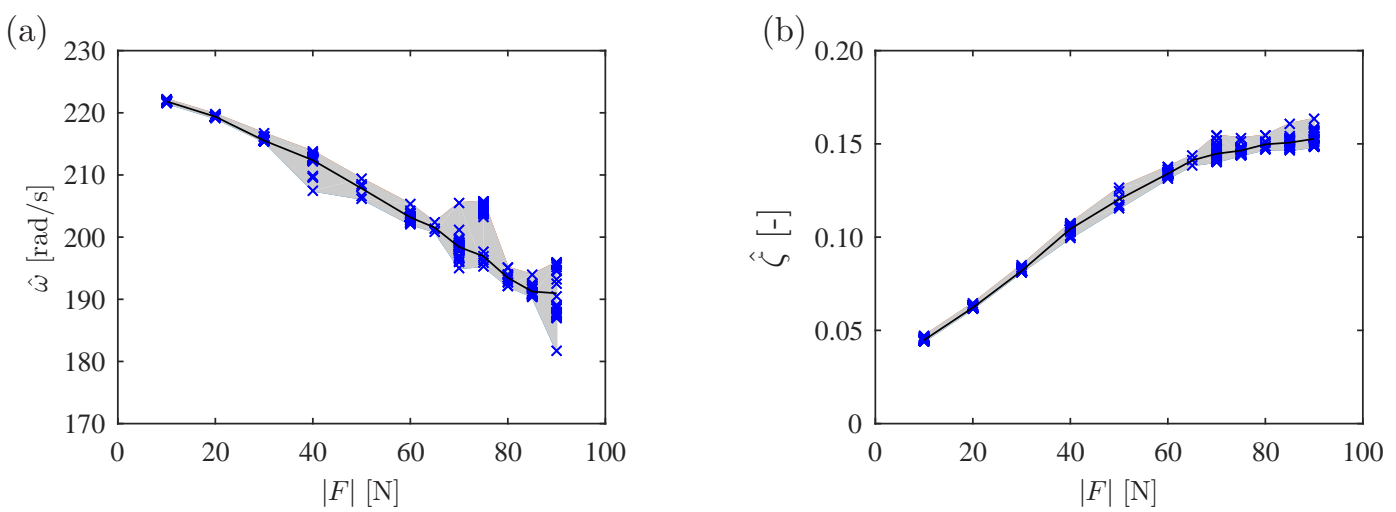

Figure 6: (a) Estimated angular natural angular frequncy and (b) damping ratio with varying excitation amplitude $|F|$. (x) identified cluster of poles, $(\bullet)$ variability given by maximum and minimum values in clusters and $(-)$ mean value of cluster. 

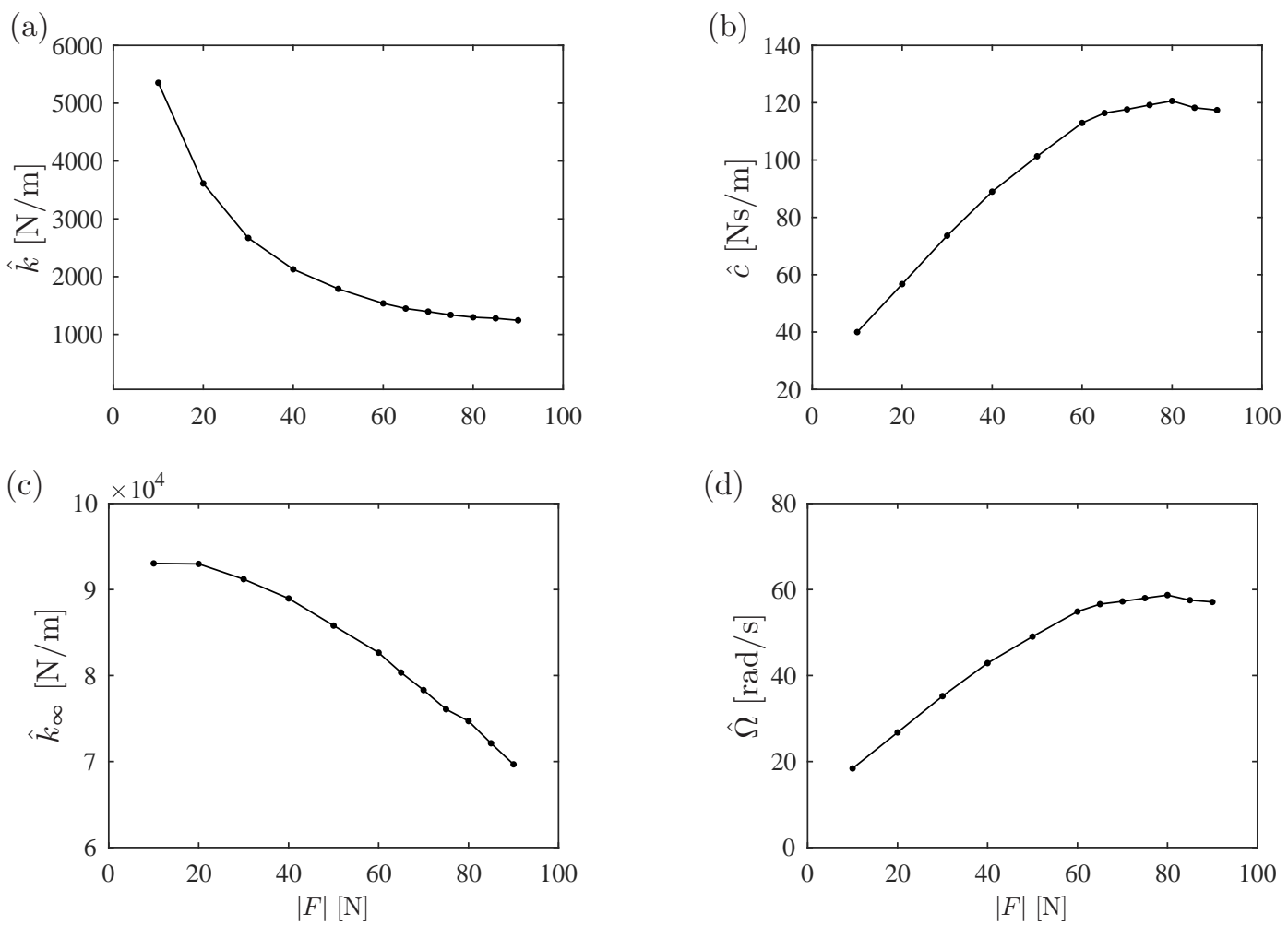

Figure 7: Estimated model parameters of the Zener model as a function of the input amplitude $A$. (a) Viscous damping $\hat{c}$, (b) linear stiffness $\hat{k}$, (c) high frequency stiffness $\hat{k}_{\infty}$ and (d) corner frequency $\hat{\Omega}$.

damping ratio in Figure 6 (b) increases with increasing excitation amplitude. The blue crosses in (a) and (b) represent the estimated natural frequency and damping ratio computed for each complex valued pole contained (as blue crosses) in the stabilized cluster, as in Figure 3 (b). The maximum and minimum values

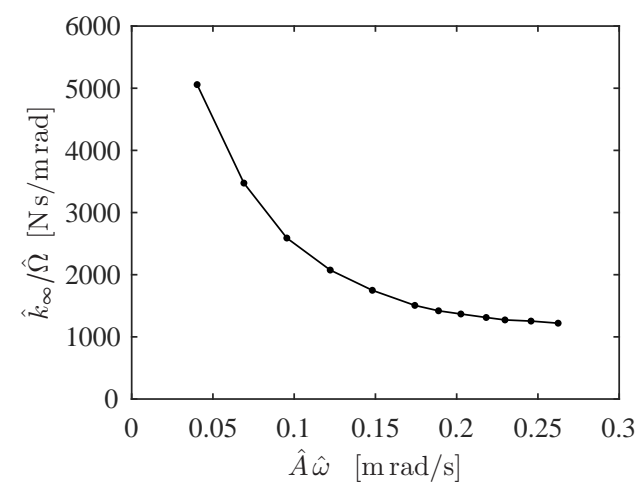

Figure 8: Response of the exact Bouc-Wen system (-) and the reconstructed response from the identification (-). (a) Excitation amplitude level of $20 \mathrm{~N}$ and (b) $80 \mathrm{~N}$. 
of the natural frequencies and damping ratios in each cluster quantify the corresponding variation of the magnitude, which is marked by the grey solid hatch in Figure 6. In both (a) and (b) it is seen that the magnitude of the variation increases with increasing excitation amplitude. The mean value of the parameter estimated in each cluster is represented by the black solid line, where the upper cluster at $75 \mathrm{~N}$ is excluded in the computation of the mean of the natural frequency, see Figure 6 (a). The two clusters formed at $75 \mathrm{~N}$ in (a) are most likely associated with asymmetries in the spectrum at resonance, and hence two estimates of stabilized frequencies are obtained at approximately $196.42 \mathrm{rad} / \mathrm{s}$ and $205.50 \mathrm{rad} / \mathrm{s}$.

Figure 7 shows that the stiffness $\hat{k}$ in (a) decreases exponentially with excitation amplitude, whereas the damping coefficient $\hat{c}$ in (b) increases to a certain magnitude and then remains nearly constant. The high frequency stiffness $\hat{k}_{\infty}$ in (c) also decreases, while the corner frequency in (d) increases and reaches a maximum magnitude, similar to the damping coefficient. Thus stiffness degradation occurs as expected from the expression in (19) because $\beta \delta<0$ in the present benchmark data. Lastly, the linear parameter $\hat{k}_{\infty} / \hat{\Omega}$ is illustrated in Figure 8 as a function of the displacement amplitude and the natural frequency, $A \omega$. This shows that $\hat{k}_{\infty} / \hat{\Omega}$ decreases with increasing $A \omega$, as it is expected from the expression in (13).

The reconstructed and exact response of the Bouc-Wen model at an excitation level of $20 \mathrm{~N}$ and $80 \mathrm{~N}$ are given in Figure 9 (a) and (b) respectively. In Figure 9 (a) the reconstructed response is seen to have a relatively low error, while in 9 (b) the error is larger, in particular when the amplitude reaches its maximum magnitude. The normalized error of the reconstructed time series is given in Figure 10 for a range of excitation levels. The normalized error is computed as previously described in Section 4. The amplitude related parameter $A$ is chosen such that the error in Figure 10 given by the red line corresponds to $A=\sigma_{x}$, the black line corresponds to $A=2 \sigma_{x}$, while the blue line is for $A=\sigma_{x} / 2$. The minimum error is obtained for $A=\sigma_{x}$. It is seen in Figure 10 by the red line that the prediction accuracy of the identification deteriorate when the excitation level increases, possibly due to an increase in the model complexity. A linear variation in the error is indicated by the error of $2.2 \%$ at $5 \mathrm{~N}$ reaching $3.6 \%$ at $95 \mathrm{~N}$. The magnitude of the error is comparable to the error reported in [12], which requires information of the system excitation signal in its identification procedure.

Due to the stochastic nature of the present random excitation, a general understanding of the performance of the identification procedure requires an analysis of numerous response time histories. In such analysis it is important to obtain long time histories, such that the variance error of the correlation function estimator in (27) is sufficiently reduced. Furthermore, improved performance of the identification method may be obtained by tuning the user-specified threshold values $d \omega_{h, j}, d \zeta_{h, j}$ and $d r_{h, j}$ in (30) and (31).

The linearized model is useful for the understanding of the dynamic response of a number of engineering systems. It is applicable in online control problems, identification from experimental testing, and updating and optimization of numerical models in structural dynamic analysis. However, when substituting a nonlinear 

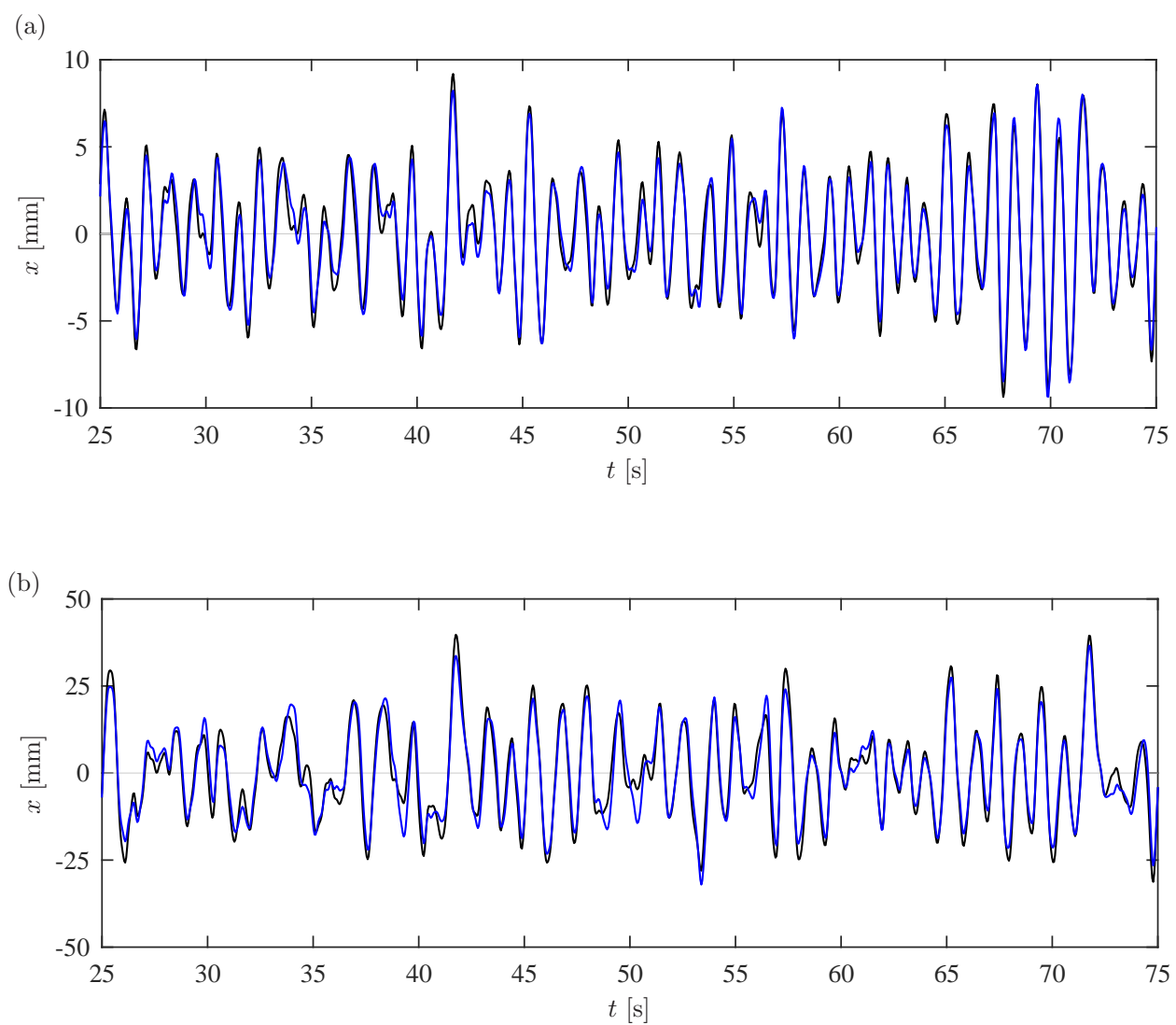

Figure 9: Response of the exact Bouc-Wen system (-) and the reconstructed response from the identification (-). Excitation amplitude level of (a) $20 \mathrm{~N}$ and (b) $80 \mathrm{~N}$.

system with an equivalent linear system whose parameters depend on the level of excitation, care must be taken when predicting parameters at extreme response levels. This is because the equivalent linear model is

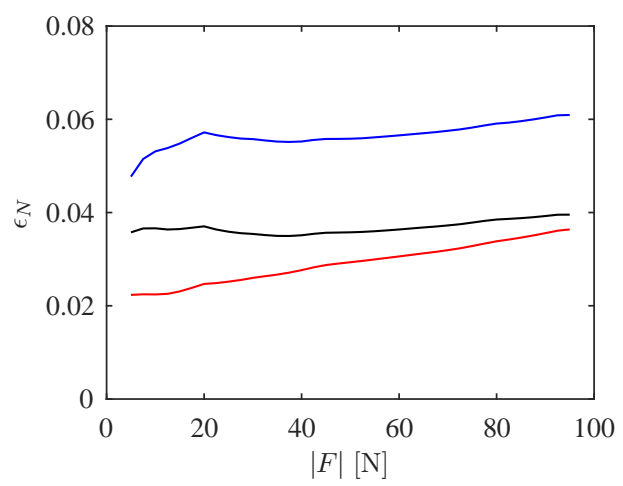

Figure 10: Norlamlized error $\epsilon_{N}$ of the reconstructed and exact reponse of the Bouc-Wen system with increasing excitation amplitude $|F|$. Reponse amplitude in the identification procedure by $(-) A=\sigma_{x},(-) A=2 \sigma_{x}$ and $(-) A=\sigma_{x} / 2$. 
inherently limited to predictions based on the first and second moments of the response. Alternatively, the extreme response may be predicted by the Fokker-Plank-Kolmogorov theory instead. However, this often implies an added computational cost and the applicability is therefore of limited use in the design phase, in which the influence of parameters on the overall response level can be obtained at a lower cost by equivalent linear models.

\section{Conclusions}

Identification of the Bouc-Wen parameters using simulated data indicates that the proposed output-only identification technique is suitable for systems represented by single-degree-of-freedom oscillators where hysteretic effects can be observed. The method assumes that the output is a stationary process and that the mass of the system is known. The identification is performed in state space, where the hysteretic system model is substituted by a derived equivalent linear relaxation damping model. The parameters of the equivalent linear model have been robustly identified at low- and high-levels of excitation amplitudes, by identifying the poles of the system by the covariance driven stochastic subspace identification technique. The identification method relies on the estimation of the complex poles and real valued poles associated with the vibratory system, which are selected for a specified threshold value and model order. The equivalent linear model assumes that the response motion resembles a sinusoid with slowly varying amplitude and phase. Therefore the method is limited to a narrow-banded response.

\section{References}

[1] K. J. Åström and T. Bohlin. Numerical identification of linear dynamic systems from normal operating records. In PH Hammond: Theory of Self-Adaptive Control Systems, Plenum Press, (1966), 96-111.

[2] P. Van Overschee and B. De Moor. N4SID: Subspace algorithms for the identification of combined deterministic-stochastic systems. Automatica, 30(1), (1994), 75-93.

[3] J. Schoukens, R. Pintelon, G. Vandersteen and P. Guillaume. Frequency-domain system identification using non-parametric noise models estimated from a small number of data sets. Automatica, 33(6), (1997), 1073-1086.

[4] B. Peeters and G. De Roeck. Reference-based stochastic subspace identification for output-only modal analysis. Mechanical systems and signal processing, 13(6), (1999), 855-878.

[5] Noël JP and M. Schoukens. Hysteretic benchmark with a dynamic nonlinearity. Workshop on Nonlinear System Identification Benchmarks, Brussels, Belgium, April 25-27, (2016), 7-14,. 
[6] B. F. Spencer, S. J. Dyke, M. K. Sain and J. Carlson. Phenomenological model for magnetorheological dampers. Journal of engineering mechanics, 123(3), (1997), 230-238.

[7] V. Hassani, T. Tegoeh and N. D. Thanh. A survey on hysteresis modeling, identification and control. Mechanical systems and signal processing, 49(1), (2014), 209-233.

[8] K. Worden and G. Manson. On the identification of hysteretic systems. Part I: Fitness landscapes and evolutionary identification. Mechanical Systems and Signal Processing, 29, (2012), 201-212.

[9] K. Worden and W. E. Becker. On the identification of hysteretic systems. Part II: Bayesian sensitivity analysis and parameter confidence. Mechanical Systems and Signal Processing, 29, (2012), 213-227.

[10] K. Worden and J. J. Hensman. Parameter estimation and model selection for a class of hysteretic systems using Bayesian inference. Mechanical Systems and Signal Processing, 32, (2012), 153-169.

[11] G. A. Ortiz, D. A. Alvarez and D. Bedoya-Ruiz. Identification of Bouc-Wen type models using the Transitional Markov Chain Monte Carlo method. Computers \& Structures, 146, (2015), 252269.

[12] J. P. Noël, A. F. Esfahani, G. Kerschen and J. Schoukens. A nonlinear state-space approach to hysteresis identification. Mechanical Systems and Signal Processing, 84, (2017), 171-184.

[13] R.E. Kalman. A new approach to linear filtering and prediction problems. Journal of Basic Engineering, 82(1), (1960), 3545 .

[14] E. Reynders. System identification methods for (operational) modal analysis: review and comparison. Archives of Computational Methods in Engineering, 19(1), (2012), 51-124.

[15] J. Ching and J. L. Beck. Real-time reliability estimation for serviceability limit states in structures with uncertain dynamic excitation and incomplete output data. Probabilistic engineering mechanics, 22(1), (2007), 50-62.

[16] K. Erazo and E. M. Hernandez. A model-based observer for state and stress estimation in structural and mechanical systems: Experimental validation. Mechanical Systems and Signal Processing, 43(1), (2014), 141-152.

[17] D. Bernal. Kalman filter damage detection in the presence of changing process and measurement noise. Mechanical Systems and Signal Processing, 39(1), (2013), 361-371.

[18] F. Naets, R. Pastorino, J. Cuadrado and W. Desmet. Online state and input force estimation for multibody models employing extended Kalman filtering. Multibody System Dynamics, 32(3), (2014), 317-336. 
[19] F. Naets, C. Javier and D. Wim. Stable force identification in structural dynamics using Kalman filtering and dummy-measurements. Mechanical Systems and Signal Processing, 50, (2015), 235-248.

[20] S. E. Azam, E. Chatzi and C. Papadimitriou. A dual Kalman filter approach for state estimation via output-only acceleration measurements. Mechanical Systems and Signal Processing, 60, (2015), 866-886.

[21] S. E. Azam, E. Chatzi, C. Papadimitriou and A. Smyth. Experimental validation of the Kalman-type filters for online and real-time state and input estimation. Journal of Vibration and Control, 23(15), (2017), 24942519.

[22] A. W. Smyth, S. F. Masri, A. G. Chassiakos and T. K. Caughey TK. On-line parametric identification of MDOF nonlinear hysteretic systems. Journal of Engineering Mechanics, 125(2), (1999), 133-142.

[23] J. W. Lin, R. Betti, A. W. Smyth and R. W. Longman. On-line identification of non-linear hysteretic structural systems using a variable trace approach. Earthquake engineering and structural dynamics, 30(9), (2001), 1279-1303.

[24] E. N. Chatzi, A. W. Smyth and S. F. Masri. Experimental application of on-line parametric identification for nonlinear hysteretic systems with model uncertainty. Structural Safety, 32(5), (2010), 326-337.

[25] F. Naets, J. Croes and W. Desmet. An online coupled state/input/parameter estimation approach for structural dynamics. Computer Methods in Applied Mechanics and Engineering, 283, (2015), 1167-1188.

[26] D. J. M. Fallais, S. Voormeeren and E. Lourens. Vibration-based Identification of Hydrodynamic Loads and System Parameters for Offshore Wind Turbine Support Structures. Energy Procedia, 94, (2016), 191-198.

[27] S. J. Elliott, M. Ghandchi Tehrani and R. S. Langley. Nonlinear damping and quasi-linear modelling. Phil. Trans. R. Soc. A, 373(2051), (2015), 20140402.

[28] Y. Wen. Method for random vibration of hysteretic systems. ASCE Journal of the Engineering Mechanics Division, 102(2), (1976), 249263.

[29] R. Bouc. Forced vibrations of a mechanical system with hysteresis. In Proceedings of the 4 th Conference on Nonlinear Oscillations, Prague, Czechoslovakia, (1967).

[30] F. Ikhouane and J. Rodellar. Systems with Hysteresis: Analysis, Identification and Control using the Bouc-Wen Model. John Wiley \& Sons, Chichester, United Kingdom, (2007).

[31] M. Ismail, F. Ikhouane and J. Rodellar. The hysteresis Bouc-Wen model, a survey. Archives of Computational Methods in Engineering, 16, (2009), 161188. 
[32] T. K. Caughey. Equivalent linearization techniques. The Journal of the Acoustical Society of America, 35(11), (1963), 1706-1711.

[33] Y.K. Wen. Equivalent linearization for hysteretic systems under random excitation. Journal of Applied Mechanics, 47(1), (1980), 150-154.

[34] J.B. Roberts and P.D. Spanos. Random vibration and statistical linearization. Courier Corporation, (2003).

[35] J. L. Beck and P. C. Jennings. Structural identification using linear models and earthquake records. Earthquake Engineering 83 Structural Dynamics, 8(2), (1980), 145-160.

[36] N. M. Krylov and N. N. Bogoliubov. Introduction to Non-Linear Mechanics. Princeton University Press, Vol.11, (2016).

[37] R. N. Iyengar. Higher order linearization in non-linear random vibration. International journal of nonlinear mechanics, 23(5-6), (1988), 385-391.

[38] C. Zener. Elasticity and Anelasticity of Metals. University of Chicago Press, Chicago, (1948).

[39] E. Reynders, J. Houbrechts and G. De Roeck. Fully automated (operational) modal analysis. Mechanical Systems and Signal Processing, 29, (2012), 228-250.

[40] E. Neu, F. Janser, A. A. Khatibi and A. C. Orifici. Fully Automated Operational Modal Analysis using Multi-Stage Clustering. Mechanical Systems and Signal Processing, 84, (2017), 308-323.

[41] Bajrić A, Høgsberg J and Rüdinger F. Evaluation of damping estimates by automated Operational Modal Analysis for offshore wind turbine tower vibrations. Renewable Energy, (2017).

[42] S. Adhikari. Dynamics of nonviscously damped linear systems. Journal of engineering mechanics, 128(3), (2002), 328-339.

[43] S. Adhikari. Qualitative dynamic characteristics of a non-viscously damped oscillator. In Proceedings of the Royal Society of London A: Mathematical, Physical and Engineering Sciences, 461, (2005), 22692288. 